\title{
Confiabilidad por el método test retest del Barómetro de la Familia versión peruana
}

\section{Reliability by test retest method of the Peruvian version of Family Barometer}

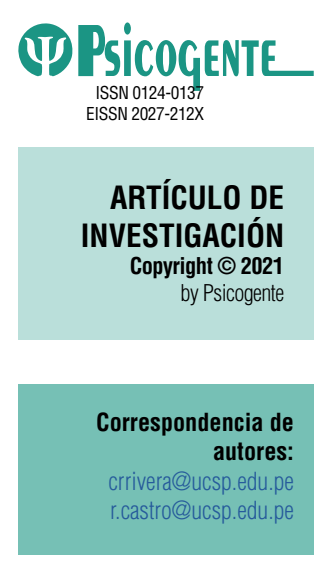

Recibido: 27-04-20 Aceptado: 20-05-20 Publicado: $30-01-21$

\author{
Renzo Rivera (iD) - Rodolfo J. Castro \\ Universidad Católica San Pablo, Arequipa, Perú
}

Resumen

Objetivo: Determinar la confiabilidad del Barómetro de la Familia por medio del método test retest.

Método: Estudio de tipo instrumental, se evaluó a 299 personas que vivían con sus parejas y/o hijos al momento de ser evaluados, el $83,6 \%$ de la muestra fueron mujeres. El rango de edad fluctuó entre los 18 y 65 años ( $M=40,7$ años). El Barómetro de la Familia (versión peruana) está compuesto por 98 ítems con diferentes opciones de respuesta (dicotómico, Likert, respuesta múltiple nominal y abierto de respuesta numérica) que evalúan la estructura y la dinámica familiar, además de la percepción que se tiene sobre temas relacionados a la familia como el aborto o el matrimonio. Se aplicaron los estadísticos kappa de Cohen ( $\mathrm{k}$ ) y coeficiente de correlación intraclase $(\mathrm{CCl})$.

Resultados: La mayoría de reactivos que indagan sobre la estructura, dinámica familiar, violencia y conductas de riesgo son confiables $(\mathrm{k} \geq 0,4 \circ \mathrm{CCl} \geq 0,4)$. Por otro lado, preguntas sobre la separación, matrimonio, homosexualidad y aborto no son confiables $(\mathrm{k}<0,4$ o $\mathrm{CCl}<0,4)$.

Conclusión: El cuestionario del Barómetro de la Familia demostró ser confiable en la medición de diversas características familiares en la población peruana.

Palabras clave: estructura familiar, dinámica familiar, confiabilidad, estudio instrumental

\section{Abstract}

Objective: It is to determine the reliability of the Family Barometer using the test retest method.

Method: An instrumental study, 299 people living with their partners and / or children were evaluated at the time of evaluation, $83,6 \%$ of the sample were women. The age range fluctuated between 18 and 65 years ( $M=40,7$ years). The Family Barometer (Peruvian version) is made up of 98 items with different response options (dichotomous, Likert, multiple nominal response and open numerical response) that evaluate the family structure and dynamics, in addition to the perception that one has about family related issues like abortion or marriage. Cohen's kappa statistics $(\mathrm{k})$ and intraclass correlation coefficient (ICC) were applied.

Results: Most of the items that inquire about the structure, family dynamics, violence and risk behaviors are reliable $(k \geq 0,4$ or $C C l \geq 0,4)$. On the other hand, questions about separation, marriage, homosexuality and abortion are not reliable $(k<0,4$ or ICC $<0,4)$.

Conclusion: The Family Barometer questionnaire proved to be reliable in measuring various family characteristics in the Peruvian population. 


\section{INTRODUCCIÓN}

La familia como objeto de investigación científica ha sido abordada con mayor profundidad por la comunidad académica a finales del siglo pasado, la literatura evidencia un notable incremento en la producción científica en este periodo (Cafarra, 2011). Los estudios consultados muestran cómo la familia es una institución con relevancia pública (Kampowski \& Gallazzi, 2015), social (Burgos, 2004) y política (Hertfelder, Martínez-Aedo \& Velarde, 2011) donde se generan vínculos de pertenencia y autoconciencia, los mismos que posibilitan una favorable inserción del individuo en el tejido social (Donati, 2014), se trata de un bien de primer orden que ha existido en cuanta civilización humana haya emergido en el tiempo (Wilcox, 2006). Es tal la relevancia de la familia que incluso la Organización para la Cooperación y el Desarrollo Económico ha creado una base de datos en la cual, sus diferentes estados miembros, han colocado información sobre aspectos como la estructura familiar, la familia y su posición en el mercado laboral, políticas públicas para las familias y los niños (OECD, 2020).

El estudio de la familia es complejo, y este ha sido abordado desde diferentes disciplinas, tópicos o áreas de conocimiento, las fuentes consultadas muestran resultados que vienen posibilitando una mejor comprensión de la institución familiar. Variedad de estudios se han orientado a entender la dinámica familiar interna; por ejemplo destacan las investigaciones sobre satisfacción (Sobrino, 2008), comunicación (Heard, 2007), funcionamiento de pareja (Perriaux, 2011) e integración familiar (Castro, et al., 2013). Así como trabajos que han explorado la relación de la familia con variedad de aspectos externos como: el impacto de la estructura familiar en la economía (Fagan, Kitt \& Potrykus, 2011) o sobre la pobreza (Castro, Rivera \& Seperak, 2017), la familia y su capacidad preventiva en escenarios de pobreza (Herrera, Salinas \& Valenzuela, 2011), asociación entre el nivel de ingresos y el tipo de familia (Aguirre, 2015), la familia como factor protector de violencia contra la mujer (Ruz, Larraín, Madrid \& Fernández, 2004) o de las conductas antisociales en adolescentes (Rivera \& Cahuana, 2016), relación entre tipo de familia y estado de salud de sus integrantes (Tovar \& García, 2007), su relevancia en la resiliencia de personas con discapacidad (Cahuana et al., 2019) y la familia como institución protectora de diversas patologías sociales (Alarcón, 2009; Sánchez, 2013) o psicológicas (Capaquira et al., 2020; Rivera, Arias \& Cahuana, 2018).

En tal sentido, podemos afirmar que la centralidad de la familia es indiscutible (Cafarra, 2011) y el bienestar que genera en las sociedades es signifi- 
cativo (Pliego, 2012). No obstante, son escasos los estudios en los que se hayan elaborado instrumentos que indaguen sobre la familia considerando sus diversas particularidades y tomando en cuenta el contexto de la región.

Teniendo en cuenta la importancia de la familia en la sociedad, son diversas las formas de medición de los distintos aspectos ligados a la familia. Podemos encontrar instrumentos tipo test como el FACES IV (Olson, Gorall \& Tiesel, 2006), la Escala de Clima Social Familiar (Moos, Moos \& Trickett, 1985) o el Cuestionario de Satisfacción Familiar (Olson \& Wilson, 1982) entre otros. Asimismo, también se encuentran encuestas dirigidas a describir y analizar a la familia desde diferentes aspectos. Se puede citar por ejemplo al The American Family Survey (Center for the Study of Elections and Democracy at Brigham Young University, 2018), instrumento diseñado para entender las experiencias de los estadounidenses en sus relaciones, matrimonios y familias y cómo esas experiencias se relacionan con una variedad de diferentes cuestiones políticas y sociales. Además de examinar las tendencias en las respuestas a preguntas básicas que sobre la forma en que los estadounidenses piensan en la formación de la familia, las actitudes sobre la fertilidad y los niños, las preocupaciones de los padres de los adolescentes y puntos de vista sobre el acoso sexual y el consentimiento.

En España, existe un instrumento denominado Barómetro de la Familia (Fundación Madrid Vivo, 2019), el cual analiza anualmente a las familias españolas sobre aspectos económicos, sociales; así como la dinámica familiar, crianza de hijos y opiniones sobre el uso de tecnologías y la sociedad. En Latinoamérica, concretamente en Argentina, existe otro instrumento con el mismo nombre, Barómetro de la Familia (Observatorio de la Familia, 2019), que desde el año 2017 mide la opinión pública sobre los hábitos, las relaciones, las costumbres y las conductas de las familias de la Ciudad Autónoma de Buenos Aires. Los tópicos evaluados buscan conocer la visión y percepción de los vecinos y la población fluctuante sobre temas coyunturales de familia. Cabe resaltar que la aplicación es trimestral y las preguntas se enfocan en un tema específico por cada aplicación. Es importante aclarar que si bien tanto los instrumentos de España, Argentina y el que presentamos en este estudio se llaman igual, Barómetro de la Familia, las preguntas que se realizan difieren. De forma similar, en Colombia el Instituto de la Familia de la Universidad de La Sabana (2019), realiza una encuesta de percepción sobre la Familia denominada como El Termómetro de la Familia, que consta de 45 preguntas sobre datos sociodemográficos, compromiso matrimonial, natalidad, dinámica familiar, ocupación e ingresos y sobre familia y sociedad. 
Como puede verse existen diversos instrumentos dedicados a analizar a las familias. Sin embargo, algo de lo que suelen carecer este tipo de encuestas son los estudios psicométricos dado que los ítems tienen diferentes formatos y se interpretan individualmente. Es por ello que en este estudio nos enfocamos en la determinación de la confiabilidad de un instrumento denominado Barómetro de la Familia que mide la estructura y la dinámica familiar, además de la percepción que se tiene sobre temas relacionados a la familia como el aborto o el matrimonio. La finalidad de la presente investigación es que dicho instrumento pueda ser aplicado en diversos lugares del Perú e incluso pueda ser adaptado a otros países.

\section{MÉTODO}

\subsection{Participantes}

Para este estudio se evaluaron a $\mathbf{3 0 0}$ personas que vivían junto a sus parejas y/o hijos al momento de la primera evaluación; mientras que en la segunda se logró encuestar a 299 personas. Teniendo en cuenta que este es un estudio que utiliza el test retest, se procederá a describir a los participantes que respondieron a ambas evaluaciones. La muestra estuvo constituida por un $83,6 \%$ de mujeres y $16,4 \%$ de varones. El rango de edad fluctuó entre los 18 y 65 años con una edad promedio de 40,7 años. Respecto al estado civil, el 43,6 \% de evaluados estaba casado, $31 \%$ era conviviente, 11,8 \% separado; mientras que el $13,5 \%$ restante era soltero, divorciado o viudo. En cuanto al nivel socioeconómico, el $17,4 \%$ de la muestra provenía del estrato $A / B$, el $33,4 \%$ del estrato $C$, el $28,6 \%$ del estrato $D$ y el $20,6 \%$ del estrato E. Cabe resaltar que la muestra fue seleccionada probabilísticamente utilizando un muestro aleatorio estratificado por el nivel socioeconómico de la población arequipeña tomando en cuenta los datos de la Asociación Peruana de Empresas de Inteligencia de Mercado (APEIM, 2019).

\subsection{Instrumentos}

El Barómetro de la Familia es un cuestionario compuesto por 98 preguntas con diversos formatos (dicotómico, Likert, respuesta múltiple y abierto de respuesta numérica), los cuales buscan recoger información sobre los diversos tipos de familia tomando en cuenta el estado civil de sus miembros (22 ítems) y la estructura familiar (16 ítems); además, indaga sobre la dinámica de la pareja y de la familia en sí (19 ítems). Para ello se incluyen preguntas sobre violencia familiar (7 ítems), consumo de alcohol, cigarro y drogas ( 6 ítems) y sobre planificación familiar ( 4 ítems). También se recaba información sobre la opinión que los padres de familia tienen respecto a la 
sociedad en su conjunto, sobre la familia, educación, matrimonio y homosexualidad (24 ítems). Dicho instrumento ha sido analizado por la opinión de jueces expertos en la investigación sobre la familia, los cuales aprobaron los ítems con un criterio flexible de $\mathrm{V}$ de Aiken, es decir que el límite inferior del intervalo de confianza sea mayor de 0,5.

\subsection{Procedimiento}

Para la realización del presente estudio primeramente se realizó el muestreo de los posibles participantes, estableciendo la proporción de la muestra según el nivel socioeconómico proveído por el APEIM (2019). Luego de ello se procedió a evaluar a las personas seleccionadas en sus hogares, asegurándoles el anonimato y confidencialidad de sus datos por medio de un consentimiento informado. El tiempo de evaluación fue en promedio de unos 30 minutos, en los cuales fue el evaluador quien llenó las encuestas teniendo en cuenta las respuestas orales de los participantes. Terminada la evaluación se les indicó a los participantes que en un plazo de 15 a 20 días se les volvería a evaluar. La segunda evaluación duró también 30 minutos en promedio y al final de la misma se les entregó un presente simbólico a los evaluados por su participación.

\subsection{Análisis de datos}

Para determinar la confiabilidad del instrumento se evaluó la estabilidad de las puntuaciones por medio del método de test-retest (Aldridge, Dovey \& Wade, 2017). Para ello se analizó cada ítem tomando en cuenta su tipo de respuesta con la finalidad de utilizar la medida de concordancia más adecuada. Los ítems cuantitativos calculamos el coeficiente de correlación intraclase $(\mathrm{CCl})$. Por otro lado, para el caso de los ítems de respuesta múltiple nominal utilizamos el coeficiente Kappa ( $\mathrm{k}$ ); para el caso de los ítems de escala Likert además del coeficiente Kappa original utilizamos el coeficiente Kappa ponderado $\left(\mathrm{k}_{\mathrm{p}}\right)$ por pesos de Ciccetti, basado en las distancias relativas entre las categorías de clasificación. Cabe resaltar que esta ponderación otorga un peso similar a los acuerdos y a los desacuerdos observados. Para una mayor precisión de los coeficientes Kappa se utilizó la técnica Jackknife para determinar los intervalos de confianza al $95 \%$. Siguiendo a Landis y Koch (1977) se consideró tanto para el CCI como para el coeficiente Kappa la siguiente escala de magnitud de concordancia: $\leq 0=$ pobre, 0,01-0,02 = débil; 0,21-0,40 = regular, $0,41-0,60=$ moderado, $0,61-0,80=$ sustancial y 0,81 a $1=$ casi perfecto. En el presente estudio, un $\mathrm{CCl}$ o Kappa mayor de 0,40 es considerado como aceptable; caso contrario consideramos que el ítem debe ser eliminado. Para el procesamiento de nuestros datos utilizamos los programas 
SPSS versión 25 y STATA versión 15, al cual se le añadió el macro denominado Kappa2 que nos permitió calcular los intervalos de confianza.

\section{RESULTADOS}

Al analizar la estabilidad en el tiempo de las respuestas dadas en el Barómetro de la Familia, en la Tabla 1 se observa que la gran mayoría de preguntas ligadas al estado civil de los evaluados tienen una casi perfecta concordancia ( $\mathrm{k}>0,80)$. Sin embargo, el ítem 5 e dirigido a personas casadas no muestra una correcta concordancia entre evaluaciones ( $k=0,25$; IC $95 \%[-1,63,1,63])$, un análisis posterior nos indicó que una posible causa del bajo nivel de concordancia es que solo tres evaluados respondieron a este ítem. Asimismo, los ítems $5 \mathrm{~b}$ que indaga sobre las segundas nupcias de una persona actualmente casada ( $\mathrm{k}=0,851$; IC $95 \%[0,281,1,42]$ ) y 6 e que indaga sobre las razones de separación de una anterior relación en los convivientes ( $\mathrm{k}=0,703$; IC $95 \%$ $[0,390,1,02])$ presentan límites inferiores menores a lo esperado. Por lo que sugerimos no eliminarlos de la encuesta; pero si tener cuidado al momento del análisis.

Tabla 1.

Índice de concordancia y coeficiente Kappa de las preguntas del Barómetro de la Familia agrupadas por el estado civil de la muestra

\begin{tabular}{|c|c|c|c|c|}
\hline & PREGUNTAS DEL BARÓMETRO DE LA FAMILIA & IC & KAPPA (IC95 \%) & $\begin{array}{l}\text { KAPPA PONDERADO } \\
\text { (IC95\%) }\end{array}$ \\
\hline \multicolumn{5}{|c|}{ SECCIÓN SOLTEROS/DIVORCIADOS/SEPARADOS/VIUDOS } \\
\hline $4 a$ & Principal razón por la que se separó de su pareja & 88,73 & $0,861(0,767-0,955)$ & - \\
\hline $4 c$ & Relación con ex pareja al tener el primer hijo & 95,83 & $0,938(0,868-1,01)$ & - \\
\hline $4 d$ & Calificación de la relación actual con padre/madre de sus hijos & 71,83 & $0,634(0,495-0,774)$ & $0,744(0,631-0,856)$ \\
\hline $4 \mathrm{e}$ & $\begin{array}{l}\text { Frecuencia con que los hijos ven al padre/madre que no vive en el } \\
\text { hogar }\end{array}$ & 82,86 & $0,798(0,690-0,906)$ & - \\
\hline $4 f$ & $\begin{array}{l}\text { Calificación de la relación de los hijos con el padre/madre que no vive } \\
\text { en el hogar }\end{array}$ & 73,24 & $0,652(0,513-0,791)$ & $0,740(0,618-0,862)$ \\
\hline \multicolumn{5}{|c|}{ SECCIÓN CASADOS } \\
\hline $5 a$ & Tipo de matrimonio por el que se casó & 95,2 & $0,881(0,786-0,977)$ & - \\
\hline $5 b$ & Convivencia antes de matrimonio & 94,17 & $0,878(0,789-0,967)$ & - \\
\hline $5 d$ & Se casó más de una vez & 99,11 & $0,851(0,281-1,42)$ & - \\
\hline $5 e$ & Principal razón por la que terminó su relación anterior & 50,0 & $0,25(-1,63-1,63)$ & - \\
\hline \multicolumn{5}{|c|}{ SECCIÓN CONVIVIENTES } \\
\hline $6 b$ & Hubiera preferido casarse con su actual pareja & 95,51 & $0,851(0,701-1,00)$ & - \\
\hline $6 c$ & Planes de casarse con su actual pareja & 96,59 & $0,903(0,789-1,02)$ & - \\
\hline $6 d$ & Convivencia/matrimonio con una persona diferente & 97,73 & $0,915(0,789-1,04)$ & - \\
\hline $6 e$ & Principal razón por la que terminó su relación anterior & 76,92 & $0,703(0,390-1,02)$ & - \\
\hline \multicolumn{5}{|c|}{ SECCIÓN CONVIVIENTES Y CASADOS } \\
\hline 10 & Calificación de su actual relación de pareja & 92,96 & $0,851(0,778-0,923)$ & - \\
\hline 11 & Calificación de la comunicación de pareja & 100,0 & $1(1,00-1,00)$ & - \\
\hline
\end{tabular}




\begin{tabular}{|c|c|c|c|c|}
\hline 12 & Calificación de la equidad en la relación & 100,0 & $1(1,00-1,00)$ & - \\
\hline \multicolumn{5}{|c|}{ Sección casados y convivientes con hijos } \\
\hline 13 & Toma de decisiones respecto a la crianza & 91,04 & $0,766(0,663-0,869)$ & \\
\hline 14 & Participación de la pareja en la crianza & 83,17 & $0,759(0,686-0,831)$ & $0,797(0,732-0,863)$ \\
\hline 15 & Relación con actual pareja al nacimiento del primer hijo & 95,82 & $0,936(0,898-0,973)$ & \\
\hline \multicolumn{5}{|c|}{ SECCIÓN PERSONAS CON HIJOS SIN IMPORTAR SU ESTADO CIVIL } \\
\hline 17 & Frecuencia con que los hijos colaboran en casa & 76,81 & $0,697(0,631-0,762)$ & $0,757(0,695-0,818)$ \\
\hline 18 & Satisfacción con la relación con los hijos & 81,32 & $0,574(0,469-0,679)$ & $0,587(0,485-0,689)$ \\
\hline 21 & Si los hijos viven junto a sus padres & & & \\
\hline a & Primer hijo & 94,06 & $0,794(0,698-0,889)$ & \\
\hline$b$ & Segundo hijo & 96,50 & $0,923(0,876-0,971)$ & \\
\hline c & Tercer hijo & 98,60 & $0,964(0,929-0,999)$ & \\
\hline 22 & $\begin{array}{c}\text { Conocimiento de dónde están los hijos menores de edad cuando no } \\
\text { están en casa }\end{array}$ & 98,27 & $0,962(0,921-0,998)$ & \\
\hline 23 & Adultos que pasan más tiempo con los niños & 92,30 & $0,837(0,757-0,917)$ & \\
\hline 24 & Cuidador de los niños cuando el/la evaluado/a no está en casa & 87,22 & $0,807(0,735-0,879)$ & \\
\hline
\end{tabular}

Para el caso de las preguntas dirigidas a personas casadas o convivientes, que indagan sobre la vida en pareja, dinámica familiar y actividad laboral tanto del evaluado como de su pareja, se puede observar en la Tabla 2 que la mayor parte de ellas tienen una casi perfecta concordancia $(k>0,80)$. Aunque, el ítem 28 (кр=0,139; IC 95 \% [0,079, 0,199]), ítem 29 (кр=0,290; IC $95 \%$ [0,204, 0,376]) e ítem 30 (кр=0,352; IC $95 \%$ [0,282, 0,421]) no presentan una adecuada concordancia; por lo que deben ser eliminados del cuestionario. Mientras que el ítem 27 ( $\mathrm{kp}=0,447$; IC $95 \%$ [0,363, 0,531]) al presentar un límite inferior con un valor del esperado, debe ser analizado con cautela.

Tabla 2.

Índice de concordancia y coeficiente Kappa de las preguntas del Barómetro de la Familia ligadas a la vida de pareja

\begin{tabular}{ccccc}
\hline & PREGUNTAS DEL BARÓMETRO DE LA FAMILIA & IC & KAPPA (IC95 \%) & $\begin{array}{c}\text { KAPPA PONDERADO } \\
\text { (IC95 \%) }\end{array}$ \\
\hline 27 & Frecuencia con que almorzaron o cenaron como familia en el último & 56,49 & $0,361(0,279-0,442)$ & $0,447(0,363-0,531)$ \\
28 & mes & 51,24 & $0,164(0,090-0,237)$ & $0,139(0,079-0,199)$ \\
29 & Satisfacción con la vida familiar & 42,25 & $0,229(0,153-0,305)$ & $0,290(0,204-0,376)$ \\
30 & Frecuencia con que hacen actividades como familia & 42,61 & $0,294(0,231-356)$ & $0,352(0,282-421)$ \\
31 & Frecuencia de visitas familiares & 85,82 & $0,299(0,142-456)$ & - \\
32 & Religión a la que pertenece la familia & 85,87 & $0,688(0,600-776)$ & - \\
33 & La familia practica su religión & 93,26 & $0,892(0,846-939)$ & - \\
34 & Tenencia de vivienda & 92,93 & $0,897(0,854-0,941)$ & $0,925(0,889-0,959)$ \\
$34 a$ & Nivel de estudios del evaluado & 91,73 & $0,835(0,741-929)$ & - \\
35 & El evaluado trabaja en lo que estudió & 93,66 & $0,898(0,853-944)$ & - \\
37 & Actividad laboral del evaluado & 96,67 & $0,947(0,905-0,989)$ & - \\
38 & Tipo de trabajo del evaluado & 91,11 & $0,783(0,681-0,885)$ & $0,799(0,705-0,895)$ \\
39 & Satisfacción los ingresos actuales & 84,92 & $0,538(0,386-0,691)$ & $0,552(0,401-0,702)$
\end{tabular}




\begin{tabular}{|c|c|c|c|c|}
\hline 40 & Decisión de administrar ingresos económicos & 94,38 & $0,894(0,829-0,958)$ & - \\
\hline \multicolumn{5}{|c|}{ SECCIÓN DE PREGUNTAS SOBRE LA PAREJA } \\
\hline 41 & Nivel de estudios de la pareja & 92,45 & $0,889(0,837-0,941)$ & $0,888(0,829-0,947)$ \\
\hline $41 a$ & La pareja trabaja en lo que estudió & 93,69 & $0,869(0,775-0,965)$ & - \\
\hline 42 & Actividad laboral de la pareja & 93,78 & $0,868(0,797-0,938)$ & - \\
\hline 44 & Tipo de trabajo de la pareja & 96,81 & $0,949(0,908-0,989)$ & - \\
\hline
\end{tabular}

En cuanto a las preguntas dirigidas a evaluar la violencia dentro de la familia, el consumo de alcohol, cigarro o drogas y la planificación familiar que tiene la pareja, se puede observar en la Tabla 3 que casi la totalidad de ítems tienen una casi perfecta concordancia ( $\kappa>0,80$ ). La única excepción es el ítem 56 (кp= 0,673; IC $95 \%[-0,416,1,76])$ que al poseer un límite inferior mucho menor que el esperado, debe de ser analizado con cuidado.

Tabla 3.

Índice de concordancia y coeficiente Kappa de las preguntas del Barómetro de la Familia ligadas a la violencia, consumo de alcohol y planificación familiar

\begin{tabular}{|c|c|c|c|c|}
\hline & PREGUNTAS DEL BARÓMETRO DE LA FAMILIA & IC & KAPPA (IC95 \%) & $\begin{array}{l}\text { KAPPA PONDERADO } \\
\text { (IC95\%) }\end{array}$ \\
\hline \multicolumn{5}{|c|}{ SECCIÓN VIOLENCIA FAMILIAR } \\
\hline 45 & Ha sufrido violencia verbal por parte de algún miembro de su familia & 91,84 & $0,812(0,738-886)$ & - \\
\hline 47 & Intensidad del maltrato verbal por parte de & & & \\
\hline a & Pareja & 87,10 & $\begin{array}{l}0,771(0,621 \\
-0,921)\end{array}$ & $0,734(0,604-0,930)$ \\
\hline $\mathrm{b}$ & Hijo/a & 100 & $1(1,00-1,00)$ & $1(1,00-1,00)$ \\
\hline$c$ & Otro miembro & 100 & $1(1,00-1,00)$ & $1(1,00-1,00)$ \\
\hline 48 & Ha ejercido violencia verbal contra algún miembro de su familia & 92,90 & $\begin{array}{l}0,836(0,766 \\
-0,906)\end{array}$ & - \\
\hline 50 & Ha sufrido violencia física por parte de algún miembro de su familia & 96,06 & $\begin{array}{l}0,649(0,443 \\
-0,855)\end{array}$ & - \\
\hline 52 & Intensidad del maltrato físico por parte de & & & \\
\hline a & Pareja & 100 & $1(1,00-1,00)$ & $1(1,00-1,00)$ \\
\hline$b$ & Hijo/a & 100 & $1(1,00-1,00)$ & $1(1,00-1,00)$ \\
\hline$c$ & Otro miembro & 100 & $1(1,00-1,00)$ & $1(1,00-1,00)$ \\
\hline 55 & Ha castigado físicamente a sus hijos & 90,48 & $\begin{array}{l}0,564(0,416 \\
-0,713)\end{array}$ & - \\
\hline 56 & Frecuencia de castigo físico a hijos & 95,00 & $\begin{array}{c}0,673(-0,416 \\
-1,76)\end{array}$ & $0,673(-0,416-1,76)$ \\
\hline \multicolumn{5}{|c|}{ SECCIÓN CONSUMO DE ALCOHOL, CIGARRO Y DROGAS } \\
\hline 57 & Consumo de cigarrillos en la familia & 88,34 & $\begin{array}{c}0,674(0,572 \\
-0,775)\end{array}$ & - \\
\hline 58 & Frecuencia de consumo de cigarrillos & 87,5 & $\begin{array}{c}0,812(0,665 \\
-0,959)\end{array}$ & $0,852(0,734-0,971)$ \\
\hline 59 & Consumo frecuente de alcohol en la familia & 87,76 & $\begin{array}{c}0,685(0,587 \\
-0,782)\end{array}$ & - \\
\hline 60 & Episodios de consumo excesivo de alcohol & 89,47 & $\begin{array}{l}0,826(0,688 \\
-0,964)\end{array}$ & $0,860(0,745-0,975)$ \\
\hline
\end{tabular}




\begin{tabular}{|c|c|c|c|c|}
\hline 61 & Consumo de drogas ilegales en la familia & 97,55 & $\begin{array}{c}0,474(0,084 \\
-0,864)\end{array}$ & - \\
\hline 62 & Frecuencia de consumo de drogas ilegales & 100 & $1,00(1,00-1,00)$ & $1,00(1,00-1,00)$ \\
\hline \multicolumn{5}{|c|}{ SECCIÓN PLANIFICACIÓN FAMILIAR } \\
\hline 63 & Uso de método anticonceptivo & 88,65 & $\begin{array}{c}0,751(0,669 \\
-0,833)\end{array}$ & - \\
\hline 64 & Método anticonceptivo utilizado & 88,24 & $\begin{array}{c}0,855(0,769 \\
-0,941)\end{array}$ & - \\
\hline 66 & Opinión sobre familias numerosas & 73,76 & $\begin{array}{c}0,603(0,524 \\
-0,682)\end{array}$ & - \\
\hline 67 & Conocimiento del tiempo de licencia padres para madres y padres & 80,14 & $\begin{array}{c}0,597(0,502 \\
-0,692)\end{array}$ & - \\
\hline
\end{tabular}

Para el caso de las preguntas del barómetro de la familia relacionadas a la opinión de los evaluados sobre la sociedad y la familia que se presentan en la Tabla 4, encontramos resultados divididos, ya que cerca de la mitad de las preguntas tienen una adecuada concordancia $(k>0,80)$. Sin embargo, el ítem 70 ( $\mathrm{k}=0,296$; IC $95 \%[0,221,0,371])$, ítem 71 ( $\mathrm{k}=0,354$; IC $95 \%[0,278$, $0,429]$ ), ítem 83 ( $k=0,300$; IC $95 \%[0,225,0,376]$ ), ítem 88 ( $k=0,396$; IC $95 \%$ $[0,272,0,519])$, ítem 90 ( $\mathrm{k}=0,327$; IC $95 \%[0,175,0,479])$, ítem 91c (кр= 0,399; IC $95 \%[0,305,0,493])$, ítem 91d (kp=0,397; IC $95 \%[0,294,0,500])$, ítem 91f (кр=0,394; IC $95 \%$ [0,294, 0,490]) e ítem 92a (кр=0,327; IC $95 \%$ $[0,227,0,427])$ tuvieron que ser eliminados por presentar una baja concordancia entre los dos momentos de la evaluación. Mientras que los ítems 78, $79,86,87,89,91$ a, 91b, 91e, 92b, 92c, 92d, 92e y 92f tendrán que ser analizados con cuidado, teniendo en cuenta que sus límites inferiores están por debajo de los valores esperados.

Tabla 4.

Índice de concordancia y coeficiente Kappa de las preguntas del Barómetro de la Familia ligadas a la opinión sobre la sociedad y la familia

\begin{tabular}{|c|c|c|c|c|}
\hline & PREGUNTAS DEL BARÓMETRO DE LA FAMILIA & IC & KAPPA (IC $95 \%)$ & $\begin{array}{l}\text { KAPPA PONDERADO } \\
\text { (IC } 95 \% \text { ) }\end{array}$ \\
\hline 70 & Principal problema de nuestra sociedad & 44,91 & $0,296(0,221-0,371)$ & - \\
\hline 71 & Principal reto de las familias de hoy & 50,53 & $0,354(0,278-0,429)$ & - \\
\hline 72 & Interés del gobierno por promover la familia & 82,80 & $0,634(0,541-0,728)$ & - \\
\hline 73 & Percepción del sistema educativo & 83,22 & $0,701(0,624-0,779)$ & $0,719(0,644-0,795)$ \\
\hline 74 & Beneficios de las actividades extracurriculares & 78,67 & $0,571(0,495-0,667)$ & - \\
\hline 75 & Calificación de la educación estatal básica & 83,92 & $0,694(0,613-0,776)$ & $0,713(0,634-0,791)$ \\
\hline 76 & Calificación de la educación superior & 86,48 & $0,741(0,663-0,819)$ & $0,761(0,687-0,834)$ \\
\hline 78 & $\begin{array}{l}\text { Importancia de la participación de los padres de familia en la vida } \\
\qquad \text { escolar de sus hijos }\end{array}$ & 74,48 & $0,445(0,339-0,551)$ & $0,436(0,329-0,543)$ \\
\hline 79 & $\begin{array}{l}\text { Percepción de si los hijos con hermanos tienen más ventajas que los } \\
\text { hijos únicos }\end{array}$ & 74,83 & $0,494(0,392-0,596)$ & - \\
\hline 80 & $\begin{array}{l}\text { Pertinencia de la inclusión del enfoque de género en el currículo } \\
\text { escolar }\end{array}$ & 74,13 & $0,553(0,466-0,640)$ & - \\
\hline
\end{tabular}




\begin{tabular}{|c|c|c|c|c|}
\hline 81 & Percepción de la participación del gobierno en las universidades & 68,53 & $0,496(0,410-0,583)$ & - \\
\hline $82 a$ & Mejoría de la educación básica & 80,35 & $0,626(0,543-0,709)$ & $0,623(0,539-0,707)$ \\
\hline $82 b$ & Mejoría de la educación superior & 77,11 & $0,541(0,451-0,630)$ & $0,541(0,451-0,631)$ \\
\hline 83 & Metas en la vida & 45,19 & $0,300(0,225-0,376)$ & - \\
\hline 84 & Percepción de la situación económica de Arequipa & 74,65 & $0,571(0,484-0,658)$ & $0,577(0,487-0,668)$ \\
\hline 85 & Necesidad de convivir antes del matrimonio & 83,16 & $0,639(0,546-0,732)$ & - \\
\hline 86 & Recomendación de solo convivir & 76,57 & $0,489(0,389-0,591)$ & - \\
\hline 87 & Valoración del trabajo doméstico por sociedad & 80,70 & $0,509(0,395-0,624)$ & - \\
\hline 88 & Ritmo laboral perjudica la relación padre-hijo & 77,74 & $0,396(0,272-0,519)$ & - \\
\hline 89 & Ritmo laboral perjudica la relación de pareja & 76,14 & $0,438(0,327-0,549)$ & - \\
\hline 90 & Aspectos en que debería centrarse la sociedad & 84,45 & $0,327(0,175-0,479)$ & - \\
\hline $91 \mathrm{a}$ & Matrimonio solo de varón y mujer & 68,88 & $0,407(0,304-0,511)$ & $0,419(0,316-0,522)$ \\
\hline $91 b$ & Matrimonio compromiso para toda la vida & 62,59 & $0,401(0,313-0,489)$ & $0,461(0,376-0,546)$ \\
\hline $91 \mathrm{c}$ & Matrimonio relación abierta a tener hijos & 64,69 & $0,368(0,271-0,464)$ & $0,399(0,305-0,493)$ \\
\hline 91d & $\begin{array}{l}\text { Hogar con relación estable entre ambos padres es lo mejor para el } \\
\qquad \text { niño }\end{array}$ & 69,93 & $0,413(0,309-0,515)$ & $0,397(0,294-500)$ \\
\hline $91 \mathrm{e}$ & Familia unidad fundamental de la sociedad & 69,37 & $0,407(0,304-0,509)$ & $0,426(0,326-0,525)$ \\
\hline $91 f$ & Los hijos ayudan a los padres a realizarse & 63,03 & $0,336(0,234-0,438)$ & $0,392(0,294-.490)$ \\
\hline $91 \mathrm{~g}$ & Divorcio como solución a problemas de pareja & 59,44 & $0,414(0,333-0,495)$ & $0,519(0,443-0,596)$ \\
\hline $92 \mathrm{a}$ & Acuerdo con aborto en general & 65,03 & $0,327(0,226-0,428)$ & $0,327(0,227-0,427)$ \\
\hline $92 b$ & Acuerdo con aborto en ciertas circunstancias & 59,79 & $0,372(0,286-0,459)$ & $0,462(0,379-0,545)$ \\
\hline $92 \mathrm{c}$ & Acuerdo con eutanasia & 61,54 & $0,411(0,325-0,497)$ & $0,463(0,378-0,549)$ \\
\hline $92 \mathrm{e}$ & Acuerdo con matrimonio homosexual & 69,58 & $0,473(0,381-0,565)$ & $0,509(0,414-0,604)$ \\
\hline $92 f$ & Acuerdo con adopción en parejas homosexuales & 75,17 & $0,466(0,364-0,569)$ & $0,459(0,354-0,565)$ \\
\hline
\end{tabular}

En la Tabla 5 se observa que la confiabilidad test-retest de aquellos ítems cuya respuesta es cuantitativa. La mayoría de ítems presentan un $\mathrm{CCl}$ que indica una adecuada concordancia entre las respuestas de los evaluados en ambos momentos en el tiempo $(\mathrm{CCl}>0,40)$. Por otro lado, también observamos que el ítem 26a (CCl=0,522; IC $95 \%$ [-0,862, 0,866]), ítem 26b (CCl=0,828; IC $95 \%$ $[-0,038,0,975])$ e ítem $26 \mathrm{~d}(\mathrm{CCl}=0,743$; IC $95 \%[0,347,0,898])$ presentan limites inferiores menores a los esperados por lo que sugerimos sean analizados con precaución en futuras aplicaciones del barómetro.

Tabla 5.

Coeficiente de correlación intraclase de las preguntas del Barómetro de la Familia

\begin{tabular}{|c|c|c|}
\hline & PREGUNTAS DEL BARÓMETRO DE LA FAMILIA & $\begin{array}{l}\text { COEFICIENTE DE CORRELACIÓN IN- } \\
\text { TRACLASE (IC95\%) }\end{array}$ \\
\hline $4 b$ & Tiempo que estuvieron juntos antes de separarse (separados) & $1(1,00-1,00)$ \\
\hline $5 c$ & Edad del evaluado en su primer matrimonio & $0,991(0,987-0,994)$ \\
\hline $5 f$ & Tiempo que estuvieron juntos antes de separarse (casados) & $1(1,00-1,00)$ \\
\hline $6 a$ & Edad del evaluado cuando convivió por primera vez & $0,933(0,898-0,956)$ \\
\hline $6 f$ & Tiempo que estuvieron juntos antes de separarse (convivientes) & $0,991(0,972-0,997)$ \\
\hline 16 & Edad del evaluado cuando tuvo su primer hijo & $0,938(0,921-0,951)$ \\
\hline 19 & Cantidad de hijos vivos que tiene el evaluado & $0,899(0,872-0,920)$ \\
\hline $20 a$ & Edad primer hijo & $0,994(0,993-0,996)$ \\
\hline
\end{tabular}




\begin{tabular}{ccc}
$20 b$ & Edad segundo hijo & $0,998(0,998-0,999)$ \\
$20 c$ & Edad tercer hijo & $0,999(0,998-0,999)$ \\
$20 d$ & Edad cuarto hijo & $0,983(0,963-0,993)$ \\
25 & Personas que viven en el hogar aparte de padres e hijos & $0,862(0,801-0,901)$ \\
$26 a$ & Cantidad de abuelos en el hogar & $0,522(-0,862-0,866)$ \\
$26 b$ & Cantidad de tíos en el hogar & $0,828(-0,038-0,975)$ \\
$26 c$ & Cantidad de padres en el hogar & $0,948(0,874-979)$ \\
$26 d$ & Cantidad de cuñados & $0,743(0,347-0,898)$ \\
$27 e$ & Cantidad de otras personas & $0,747(0,609-0,836)$ \\
36 & Horas que el evaluado trabaja a la semana & $0,919(0,891-0,939)$ \\
43 & Horas que trabaja la pareja a la semana & $0,875(0,834-0,906)$ \\
65 & Número ideal de hijos & $0,769(0,708-817)$ \\
68 & Cantidad de meses de descanso por maternidad & $0,688(0,599-0,757)$ \\
69 & Cantidad de meses de descanso por paternidad & $0,714(0,594-0,799)$ \\
\hline
\end{tabular}

\section{DISCUSIÓN}

En el presente estudio se analizó la confiabilidad del Barómetro de la familia por medio del método de test-retest a una muestra de mujeres y varones mayores de edad provenientes de la ciudad de Arequipa. Los resultados obtenidos sugieren que el cuestionario evaluado es confiable en sus diversas secciones, lo cual lo convierte en uno de los primeros instrumentos de su tipo en presentar indicadores psicométricos que respalden su utilización.

Al analizar el Barómetro de la Familia en sus diversas secciones se observa que las preguntas dedicadas a evaluar las características de los participantes según su estado civil presentan una adecuada concordancia a lo largo del tiempo, lo mismo para las preguntas que ahondan en la vida de pareja, dinámica familiar y actividad laboral de aquellas personas que son casadas o convenientes, lo cual es un indicador de la precisión de los reactivos. Respecto a la vida de pareja y dinámica familiar, estos resultados se asemejan a lo hallado por Hamilton et al. (2015) cuando analizaron las propiedades psicométricas del Systemic Clinical Outcome and Routine Evaluation (SCORE). En dicho estudio las dimensiones de fortalezas $(\mathrm{CCl}=0,85)$ y comunicación $(\mathrm{CCl}=0,88)$ mostraron la estabilidad de las puntuaciones en el test retest realizado. De igual forma, hay coincidencia con lo reportado por Scholte y Van der Ploeg (2015) cuando desarrollaron el Family Questionnaire (FQ), dichos autores indicaron que las dimensiones de comunicación $(\mathrm{CCl}=0,78)$, organización $(\mathrm{CCl}=0,78)$ y relación de pareja $(\mathrm{CCl}=0,89)$ presentan una adecuada confiabilidad. Aunque es importante resaltar que tanto el SCORE como el FQ solo tienen preguntas tipo Likert, lo cual genera que las respuestas sean más estables aún que las del Barómetro de la familia. Asimismo, lo hallado en esta investigación se asemeja a lo reportado por Moreno et al. (2017) cuando 
se elaboró el Barómetro de opinión de la infancia, en el cual la sección que indago sobre aspectos sociodemográficos de los niños y sus familias mostro ser válida y confiable. Cabe resaltar que dicho instrumento es similar al presentado en esta investigación por su estructura y formato de preguntas.

Por otro lado, los ítems que indagan sobre las razones de separación de pareja y sobre segundas nupcias tienden a no ser tan confiables, por lo que sus respuestas deben de ser analizadas con cuidado. En este punto nuestros resultados diferentes de lo encontrado por Hamilton et al. (2015) con el SCORE, dado que en su investigación la sección análoga a la nuestra que es dificultades obtuvo una puntuación alta en el análisis de confiabilidad $(\mathrm{CCl}=$ $0,95)$. El motivo por el cual dichas preguntas en el Barómetro de la familia no son tan estables en el tiempo puede deberse al tipo de formato que tienen, como también al hecho de que las personas consideran que la separación o las segundas nupcias son aspectos muy privados, por lo que podrían cambiar sus respuestas como una forma de proteger su intimidad. Otro punto ligado al anterior, es que los evaluados han tratado de dar respuestas que sean lo más cercano a lo socialmente aceptado, lo que mayormente se conoce como deseabilidad social (Edwards, 1957).

Para el caso de aquellos reactivos contenidos en la sección de violencia dentro de la familia y conductas de riesgo como el consumo de alcohol y drogas también se encontró un nivel adecuado de concordancia en las respuestas a lo largo del tiempo. Si bien las preguntas del presente instrumento, respecto a la violencia dentro de la familia, son muy puntuales se han obtenido coeficientes que pueden ser equivalentes a los de instrumentos mucho más especializados como el Conflict Tactics Scale (CTS-2) que contó con un coeficiente de correlación de 0,76 para el maltrato físico y 0,69 para el psicológico en un análisis test retest (Vega \& O'Leary, 2007). Lo cual es un indicador de que el Barómetro de la familia recoge la percepción real de los evaluados y permitirá obtener datos que reflejen los problemas que las familias tienen que afrontar.

En cuanto a las preguntas de la sección sobre percepción de la familia y la sociedad, se halló que la mitad de ellas tenía indicadores adecuados de confiabilidad. Sin embargo, los ítems que indagaron sobre las metas en la vida, los problemas que enfrenta la sociedad y el aborto tuvieron una confiabilidad menor a lo esperado por lo que deberían de ser analizadas con cuidado. Además que los ítems que inquirieron sobre aspectos delicados como la concepción del matrimonio, la eutanasia, el matrimonio entre personas del mismo sexo y la adopción por parte de parejas del mismo sexo tuvieron que 
ser eliminados por presentar respuestas inconsistentes. Una posible explicación sobre el porqué las preguntas antes mencionadas no son confiables es que la sociedad arequipeña, como muchas otras en América Latina, es mayormente conversadora (Gómez, 2013; Vaggione \& Mujica, 2013), por lo que como una forma de evitar la desaprobación social podrían dar respuestas diferentes en dos momentos en el tiempo.

Los ítems de respuesta cuantitativa mostraron también una adecuada estabilidad de puntuaciones a lo largo del tiempo, salvo los ítems que evalúan la cantidad de parientes que viven en el hogar los cuales presentan unos valores de confiabilidad un poco por debajo de los esperado, por lo cual deben de ser analizados con precaución.

Al ser este un estudio pionero en su tipo, no está exento de limitaciones. Una de ellas es que solo se evaluó a las familias de la ciudad de Arequipa, por lo que se sugiere que se replique la investigación en otras ciudades, ya que tienen diferentes idiosincrasias. Asimismo, el alto costo de tomar un test retest generó que no se pueda evaluar a una muestra más grande de personas. Se espera que futuras investigaciones logren confirmar la idoneidad de las preguntas formuladas en el Barómetro de la Familia para evaluar a la misma en sus diversos aspectos.

\section{CONCLUSIÓN}

Se concluye que el Barómetro de la familia versión peruana es un instrumento confiable para poder evaluar aspectos de estructura como de dinámica familiar y de pareja.

El aporte de la presente investigación radica en que se pone a disposición de la comunidad académica un instrumento que permitirá llevar a cabo estudios tanto descriptivos como inferenciales de la realidad que afrontan las familias no solamente en el Perú, sino también en otros países de Latinoamérica, una vez sea adaptado a dichas realidades. Asimismo, aporta metodológicamente con la estrategia a seguir para poder calcular la confiabilidad de un instrumento que tenga ítems de diferentes escalas de medición, lo cual conllevará que otros investigadores también puedan realizar estudios similares.

Se recomienda que en futuras investigaciones se trate de evaluar a muestras más grandes. Además que se tome en cuenta la de diferentes ciudades, lo que permitirá generar una mejor descripción de las familias dentro de un país determinado. 
Conflictos de interés: Los autores declaran que no existen conflictos de ningún tipo en la realización de esta investigación ni en la redacción de este manuscrito.

Financiamiento: El presente estudio fue financiado por la Universidad Católica San Pablo.

Agradecimientos: A la Universidad Católica San Pablo, por haber financiado y haber hecho factible la creación y aplicación de un instrumento que permita comprender mejor la realidad de la institución familiar.

\section{REFERENCIAS}

Aguirre, M. (2015). El Impacto de la Familia en la Economía. Una Propuesta para Lograr un Desarrollo Sostenible. Guatemala: FADEP. Disponible en https://sophiaaguirre.squarespace.com/

Alarcón, R. (2009). Psicología de la felicidad. Lima: Universidad Ricardo Palma.

Aldridge, V. K., Dovey, T. M. \& Wade, A. (2017). Assessing test-retest reliability of psychological measures: persistent methodological problems. European Psychologist, 22(4), 207-218. https://doi.org/10.1027/1016-9040/a000298

APEIM. (2019). Niveles socioeconómicos 2018. http://apeim.com.pe/wp-content/ uploads/2019/11/APEIM-NSE-2018.pdf

Burgos, J. (2004). Diagnóstico sobre la familia. Madrid: Biblioteca Palabra.

Caffarra, C. (2011). La familia: Un lugar de la experiencia de comunión. Persona y Cultura. (9), 69-79.

Cahuana, M., Arias, W., Rivera, R., \& Ceballos, K. (2019). Influencia de la familia sobre la resiliencia en personas con discapacidad física y sensorial de Arequipa, Perú. Revista Chilena de Neuro-Psiquiatria, 57(2), 118-128. https://doi.org/10.4067/ S0717-92272019000200118

Capaquira, J., Arias, W., Muñoz del Carpio, A. \& Rivera, R. (2020). Psychological Distress, Family Relationship and Reasons for Consultation of Women from Arequipa, Peru. Atención familiar, 27(2), 76-80. https://doi.org/10.22201/ facmed.14058871p.2020.2.75680

Castro, R., Arias, W. Domínguez, S., Masías, M., Salas, X., Canales, F. \& Flores, A. (2013). Integración Familiar y variables socio-económicas. Revista de Investigación de la Universidad Católica San Pablo, 4(4), 35-63. https://ucsp.edu.pe/ images/direccion_de_investigacion/PDF/revista2013/Integracion-familiar-y-variables-socioeconomicas.pdf

Castro, R., Rivera, R. \& Seperak, R. (2017). Impacto de la composición familiar en los niveles de pobreza de Perú. CUHSO, 27(2), 60-88. http://dx.doi.org/10.7770/ cuhso-v27n2-art1229

Center for the Study of Elections and Democracy at Brigham Young University. (2018). The American Family Survey. Recuperado el 02/06/19 de https://www. deseretnews.com/media/misc/pdf/afs/2018-AFS-Final-Report.pdf

Donati, P. (2014). La política familiar: por un welfare relacional y subsidio. Santiago de Chile: Pontificia Universidad Católica de Chile. Ediciones UC.

Edwards, A. L. (1957). The social desirability variable in personality assessment and research. New York: Dryden.

Fagan, P., Kitt, A. \& Potrykus, H. (2011). Marriage and Economic Well-Being: The Economy of the Family Rises or Falls with Marriage. Marriage Research Synthesis. (pp.1-22). USA: Marriage and Religion Research Institute. 
Fundación Madrid Vivo. (2019). Barómetro de la Familia. Recuperado el 15/04/19 de http://madridvivo.org/viii-barometro-de-la-familia/

Gómez, D. (2013). La apuesta teórica y política por las regiones desde el feminismo. En G. Cevasco (Ed.), 25 años de feminismo en el Perú: Historia, confluencias y perspectivas (pp. 57-60). Centro de la Mujer Peruana Flora Tristán. http://www2. congreso.gob.pe/sicr/cendocbib/con4_uibd.nsf/C08CBB7DF991A3FF05257B1700675D74/\$FILE/BVCI0003574.pdf

Hamilton, E., Carr, A., Cahill, P., Cassells, C. \& Hartnett, D. (2015). Psychometric properties and responsiveness to change of 15 - and 28- item versions of the SCORE: A family assessment questionnaire. Family Process, 54(3), 454-463. http://dx.doi.org/10.1111/famp.12117

Heard, H. (2007). Fathers, Mothers, and Family Structure: Family Trajectories, Parent Gender, and Adolescent Schooling. Journal of Marriage and Family, 69(2): 435-450. https://doi.org/10.1111/j.1741-3737.2007.00375.x

Hertfelder, E., Martínez-Aedo, M. \& Velarde, L. (2011). La familia, desafío para una nueva política. Propuestas para una política pública con perspectiva de familia. Madrid: Instituto de Política Familiar.

Herrera, S., Salinas, V. \& Valenzuela, E. (2011). Familia, pobreza y bienestar en Chile: un análisis empírico de las relaciones entre estructuras familiares y bienestar. Revista del Instituto de Sociología y el Centro de Políticas Públicas de la Universidad de Chile, 11(1), 1-19. https://politicaspublicas.uc.cl/wp-content/ uploads/2015/02/familia-pobreza-y-bienestar-en-chile.pdf

Kampowski, S. \& Gallazzi G. (2015). Familia y desarrollo sostenible. Arequipa: Fondo Editorial de la Universidad Católica San Pablo.

Landis J. R. \& Koch, G. G. (1977). The measurement of observer agreement for categorical data. Biometrics, 33(1), 159-174. https://doi.org/10.2307/2529310

Montero, I. \& León, O. G. (2007). A guide for naming research studies in Psychology. International Journal of Clinical and Health Psychology, 7(3), 847 - 862. https:// www.redalyc.org/articulo.oa?id $=33770318$

Moos, R. H., Moos, B. S. \& Trickett, E. (1985). Escala del Clima Social Familiar FES. Madrid: TEA Ediciones.

Moreno, C., Rivera, F., Ramos, P., Sánchez, I., Jiménez, A., García, I., Moreno, C., Paniagua, C., Villafuerte, A., Ciria, E., Abate, M. \& Morgan, A. (2017). Barómetro de opinión de la infancia: manual para su uso. Madrid: UNICEF Comité Español. https://www.unicef.es/publicacion/barometro-de-opinion-de-la-infancia-manual-para-su-uso

Observatorio de Familia. (2019). Barómetro de la Familia. Recuperado el 14/04/19 de https://www.buenosaires.gob.ar/vicejefatura/desarrollofamiliar/barometro

OECD. (2020). OECD Family database. http://www.oecd.org/social/family/database. htm

Olson, D. H., Gorall, D. M. \& Tiesel, J. (2006). FACES IV: Development and Validation. Minnesota: Life Innovations. www.facesiv.com

Olson, D. H. \& Wilson, M. (1982). Family Satisfaction. In D. H. Olson (Ed.), Families: What Makes Them Work? California: Sage Publishing

Perriaux, J. (2011). La familia ante algunos desafíos de la realidad actual. Persona y Cultura, (9), 12-33.

Pliego, F. (2012). Familias y bienestar en sociedades democráticas. El debate cultural del siglo XXI. México: Miguel Ángel Porrúa. 
Rivera, R., Arias, W. \& Cahuana, M. (2018). Perfil familiar de adolescentes con sintomatología depresiva en Arequipa. Revista Chilena de Neuro-Psiquiatria, 56(2), 117-126. https://doi.org/10.4067/s0717-92272018000200117

Rivera, R., \& Cahuana, M. (2016). Influencia de la familia sobre las conductas antisociales en adolescentes de Arequipa - Perú. Actualidades en Psicología, 30(120), 85-97. https://doi.org/http://dx.doi.org/10.15517/ap.v30i120.18814

Ruz, M., Larraín, S., Madrid, A. \& Fernández, M.(2004). Detección y análisis de la prevalencia de la violencia intrafamiliar en la región de Coquimbo. Pontificia Universidad Católica de Chile, Dirección de Estudios Sociológicos.

Sánchez, J. (2013). Evolución de la dinámica familiar en contextos de riesgo: efectos de un programa sistémico. Psicogente, 16(29), 184-196. http://revistas.unisimon. edu.co/index.php/psicogente/article/view/1950

Scholte, E., \& Van der Ploeg, J. (2015). The Family Questionnaire: A Measure to Assess the Quality of Family Functioning. Journal of Family Issues, 36(13), 1810-1828. https://doi.org/10.1177/0192513X13506707

Sobrino, L. (2008). Niveles de satisfacción familiar y de comunicación entre padres e hijos. Avances en Psicología, 16(1), 109-137. https://www.unife.edu.pe/pub/ revpsicologia/sastisfaccionfamiliar.pdf

Tovar, L. \& García, G. (2007). La producción de salud infantil en Colombia: una aproximación. Desarrollo y Sociedad, 59, 21-61. http://vitela.javerianacali.edu.co/ handle/11522/3505

Universidad de la Sábana. (2019). Termómetro de la Familia. Recuperado el 14/04/19 de https://www.unisabana.edu.co/empresaysociedad/instituto-de-la-familia/ publicaciones/termometro-de-la-familia/

Vaggione, J. M. \& Mujica, J. (2013). A modo de introducción: algunos puntis de discusión en torno al activismo (religioso) conservador en América Latina. En J-M. Vaggione \& J. Mujica (Comps.) Conservadurismo, religión y política Perspectivas de Investigación en América Latina - Las estrategias discursivas de la iglesia católica en torno a la Educación sexual. Córdova: Ferreyra editor.

Vega, E. M., \& O'leary, K. D. (2007). Test-retest reliability of the revised Conflict Tactics Scales (CTS2). Journal of Family Violence, 22(8), 703-708. https://doi. org/10.1007/s10896-007-9118-7

Wilcox, B. (2006). El Matrimonio sí importa: veintiséis conclusiones de las Ciencias Sociales. Charlottesville: Social Trends Institute.

Esta obra está bajo: Creative commons attribution 4.0 international license. El beneficiario de la licencia tiene el derecho de copiar, distribuir, exhibir y representar la obra y hacer obras derivadas siempre y cuando reconozca y cite la obra de la forma especificada por el autor o el licenciante.

\section{(cc) BY}

